\title{
Electrostatic/entropic macromolecule manipulation in nanochannel. Swapping of macromolecule locations
}

\author{
Waldemar Nowicki ${ }^{1}$
}

Received: 1 July 2019 / Accepted: 14 August 2019 / Published online: 24 August 2019

(C) The Author(s) 2019

\begin{abstract}
Evolution of a polyion and an electrically uncharged macromolecule in confined environment, represented by a tight nanopore, induced by external electric field, is investigated. The motion of the uncharged macromolecule is forced by the polyion. In suitably selected conditions, the collision between macromolecules, compression of the uncharged macromolecule, and finally the swap of positions of macromolecules in the nanochannel are observed by means of the nonequilibrium Monte Carlo method on the lattice. The exemplary trajectories of macromolecules, their geometry, and energetics are analyzed. The dependence of the time of swapping on the number of macromolecule beads is described by scaling laws.
\end{abstract}

Keywords Molecular modeling $\cdot$ Monte Carlo simulation $\cdot$ Polyelectrolytes

\section{Introduction}

The single-macromolecule manipulations have been of growing interest in the last two decades due to the development of new techniques allowing specific operations like trapping, moving, and sorting of particular objects in the nanoscale systems [1]. The manipulation techniques are based on a variety of physical laws and inventions [1,2]. They include the glass microfiber micromanipulation [3], the magnetic beads used as the force probes controlled by optical microscopy (magnetic tweezers) [4], the atomic force microscopy allowing measurements of mechanic properties of single macromolecule such as the force needed for stretching its conformation or for the rupture of a covalent bond $[5,6]$, and the optical tweezers which can catch and drag nanosize dielectric objects, and also some macromolecules [7]. These techniques have been developed mainly for biophysical applications, but their use has quickly spread to other domains, such as the study of Brownian motors [8]; untangling, unknotting, and unlinking of DNA molecules [1]; sorting of microscopic particles by size or by refractive index [9]; and microrheological measurements in small volumes and on short length scales,

Waldemar Nowicki

gwnow@amu.edu.pl

1 Faculty of Chemistry, Adam Mickiewicz University in Poznań, ul. Uniwersytetu Poznańskiego 8, 61-614 Poznań, Poland including the interior of cells and prestretching and stabilizing of DNA before threading through a nanopore [10]. These very advanced methods can be supplemented by innovative applications of electrokinetic phenomena, i.e., electrophoresis [11] and electroosmosis [12] often assisted by optical effects such as optoelectronic tweezers [13], optically induced dielectrophoresis [14], methods based on thermally induced gradients of permittivity and conductivity of fluids $[15,16]$, alternating current electroosmosis [17, 18], optically induced electrokinetic effect [17], and dielectrophoresis [19] to list a few. The nonzero energy of interaction between polymer beads and walls of the tight slit can produce jumps of macromolecule from one surface to the other [20].

Spontaneous motion of macromolecules can be also induced by the particular geometry of tight cavities. This motion - the entropophoresis - is an emerging nanofluidic method using nanoscale confinement to control the spontaneous transport and separation of macromolecules and other nanoscale objects [21]. The free energy landscapes of macromolecules in tight confinement [22] can be designed to entropically manipulate their random diffusion, e.g., the asymmetry of 3-branched star macromolecule can induce its spontaneous motion [23].

Recent progress in chemical modification and technology of material conversion having a molecular-scale porous structure with well-controlled pore diameters and interlayer distances has led to construction of a variety of tight nanocavities including complex channels, slit matrices, or labyrinths, in which motions and locations of particular species can be 
controlled. An example is a graphene oxide membrane [24-28], for which high-speed water flow [29] and ion transports [30] across nanochannels and nanoslits stacking sheets have been observed. Such a transport of ions or polyions can be controlled using voltage-gated channels [31,32].

Combinations of the methods mentioned above have opened a new approach to the application of thermodynamically controlled processes to automate the operation of lab-ona-chip technology, for both practical applications and fundamental investigation [21]. Such methods would allow controlled displacement of individual macromolecules or polyions, their forced translocation in nanopores and ionswitched nanochannels, and their segregation or trapping. Nevertheless, the physical backgrounds of certain techniques are still not fully understood.

A very interesting method for micromanipulation of macromolecules is a superposition of two effects - the external electrostatic field and the tight nanoscopic confinement, leading to the electrophoretic and entropophoretic effects, especially in systems containing a pair of uncharged macromolecules and/or macromolecules bearing functional ionogenic groups. An example of the phenomena occurring in such systems is the spontaneous segregation of genetic material, chromosomes, after replication accompanying the cell division in some bacteria [33]. This entropy-driven process seems to be strongly influenced by electrostatic interactions in the electrolyte solution [34].

Polymer molecules under nanoconfinement show unique dynamical properties different from those of free macromolecules in the bulk [35], altered by both the statics [36] and dynamics of a polymer chain $[37,38]$. Some approaches to the description of chains in nanoconfinement are based on the excluded volume effects and elasticity of chains (both governed by conformational entropies of chains) influence on their behavior. The models concern SAW (self-avoiding walk) chains with different stiffness and confinement limits [39-41]. Numerous studies have been performed on segregation of macromolecules [42, 43], polymer translocation through narrow pores $[44,45]$ and chamber-pore system [46], prestretching and stabilizing of DNA before threading through a nanopore [10,47], etc.

The main idea of the current study is to use one of the macromolecules - a polyion - as a tool for micromanipulation of the position of another one - an electrically neutral macromolecule, by means of electrophoretic and entropophoretic effects. The study concerns the tight nanoconfinement represented here by a nanochannel. Thus, the objective of the study is to perform a numerical experiment in attempt to answer the question whether the macromolecule endowed with electric charge can be used for induction of controlled movement of the electrically neutral one and which mutual interactions between macromolecules will be observed, taking into account the possibility of translocation, interpenetration/segregation of macromolecules, or their swapping. The scheme of processes of potential interest is shown in Fig. 1. The paper concentrates on the swapping process; however, other processes shown in the figure are also observed in the study.

The paper is organized as follows: in "Model and simulation method," the details of the simulation technique applied are described including the model representation of polymer chains, simple electrolyte ions, and geometry of the systems. The results of single simulation and the analysis of evolution of geometric and thermodynamic properties of migrating chains are gathered in "Details of motions of the polyion and uncharged macromolecule." Finally, some generalizations based on formulation of some scaling laws describing the influence of chain bead number and the nanochannel width are presented in "Generalization of the system behaviorsome scaling laws."

\section{Model and simulation method}

The study was performed by means of simply and relatively fast nMC (nonequilibrium Monte Carlo) method [48]. The method was chosen because of the ease of its implementation.

The simulations were performed on the three-dimensional cubic lattice of the lattice constant $b$. The confined space of the studied process was a rectangular nanochannel with one end open and the other end closed. The length of the nanochannel was $600 b$ and the width $D$ was equal from $3 b$ to $11 b$ (depending on the simulation, in most simulations $D=5 b$ ). The choice of geometry of the nanochannel was determined by the fact that the simulation performed in the nanochannel with both ends open lasted very long as a result of pushing of neutral macromolecule by polyion over long distances.

The polymer chains are represented by SAWs embedded in the lattice. Each monomer is identified by the site on the lattice (B bead), so each chain is an ensemble of $N$ consecutive sites occupied by B beads. The beads succeeding along the chain are located in adjacent lattice sites at the distance $b$. Two different polymer chains were considered: one (C1) made of two types of beads: electrically neutral and bearing elementary electric charge, and the other (C2) consisting only of electrically uncharged beads located at equal distances along the chain. A single charged bead, bearing an electric charge $q_{\mathrm{P}} / e$ equal to +1 (where $e$ is the elementary charge), was assumed to interact electrostatically with all other charges in the system. Since the general athermal conditions were assumed, all other intermolecular interactions were neglected, except the excluded volume effects. Both studied chains consisted of $N$ beads. The simulations were performed for a constant electric charge of the polyion determined by the constant density of electrically charged beads equal to $\sigma$ (the ratio of the number of charged beads to the total number of beads in the chain, usually $\sigma=0.2$ ). Positions of charged beads along the chain were 
Fig. 1 Scheme of mutual motions of positively charged polyion (C1) and uncharged macromlecule $(\mathrm{C} 2)$ in the electrostatic field: a) motion of $\mathrm{C} 2$ forced by $\mathrm{C} 1, \mathbf{b})$ interpenetration of $\mathrm{C} 1$ and $\mathrm{C} 2$ and $\mathbf{c}$ ) capturing of $\mathrm{C} 1$ in the end of nanochannel and free diffusion of $\mathrm{C} 2(N=200)$

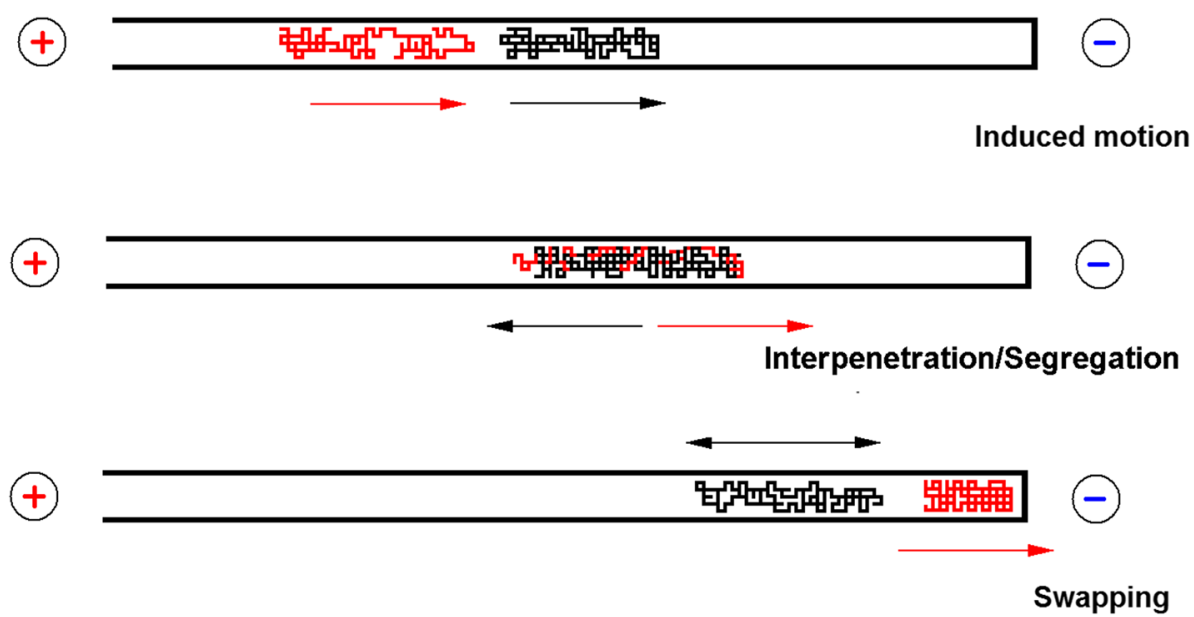

b) fixed. The nearest neighboring lattice sites of these beads were assumed to be unavailable to the other charged beads, mimicking thus the ion solvation effect. Simulations were performed for the SAW chains using the Verdier-Stockmayer algorithms [49, 50].

The dispersion medium was represented by a solution of monovalent electrolyte molecules. Ions were treated as lattice nodes containing the electric charges $q_{\mathrm{I}} / e$ equal to -1 or +1 . Individual ions (I beads) occupied lattice nodes surrounded by empty nodes corresponding to solvent molecules constituting the ion solvation shell. The lattice sites forming the solvation shell were assumed to be unavailable to charged beads. This mode of accounting for ion solvation, similar to solvation of polymer-charged beads, is similar to that proposed by Ruckenstein et al. [51]. The assumed excluded volume around a single charged bead corresponded roughly to that estimated by Paunov et al. [52] to account for the hydration of ions, which is six times the volume of the ion. The shell thickness exceeded the Bjerrum length for the relative dielectric permittivity of the medium used in the study $(\varepsilon=80)$, thus allowing the neglect of the Manning condensation [53]. The adopted value of $\varepsilon$ for corresponds to the aqueous bulk phase.

Numbers of positively and negatively charged beads I in the simulation box ( $n_{+}$and $n_{-}$respectively) were determined by the ionic strength $I_{\mathrm{S}}$ of the solution which was defined as:

$I_{\mathrm{S}}=\frac{1}{2}\left(\frac{\left(n_{+}+n_{-}\right) q_{\mathrm{I}}^{2}}{e^{2} V}\right)$

where $V$ represents the volume of the microchannel. The numbers $n_{+}$and $n_{-}$were calculated taking into account the charges of the polymer to ensure electroneutrality. The initial positions of beads I were random as generated by using a pseudorandom number generator, taking into consideration the effects of the excluded volume of the chains and previously randomly generated I beads together with their solvation shells.

Relaxation of the system was achieved by random micromodifications of positions of beads I made with steps that have a length $b$ in any lattice direction. Numbers of charged beads I in the simulation box were adjusted by assuming the ionic strength $\left(I_{\mathrm{S}}=0.001\right)$ and the electric charge carried by polyion $\mathrm{C} 1$.

At both ends of the channel, the periodic boundary conditions were applied for the motion of simple ions. Since in the course of the simulation, the ions move to the opposite charged electrodes outside of the box, the boundary conditions guarantee the electroneutrality of the system. At the closed end, the membrane impermeable for macromolecules but permeable for simple ions was applied. The model membrane mimics the real semipermeable membrane or the electrode surface on which the ion creation and annihilation by the electrode reactions take place. There are no additional constraints applied on the other end of the nanochannel since both types of macromolecules do not cross it: $\mathrm{C} 1$, because it moves in the opposite direction; and C2, because the simulation stops before reaching the nanochannel end.

The external electric field $E$ was applied in the direction parallel to the nanochannel. Its polarization forced the motion of $\mathrm{C} 1$ chain towards the closed end and motions of ions in directions specified by their signs. Initial locations of both chains are shown in Fig. 1a.

In the course of simulation, the components of the free energy of the system were calculated. Since the internal energy of nonionic intra- and intermolecular interactions for the polymer chains was equal to zero as a result of the assumption of athermal conditions, the only energy component of the system was the energy of electrostatic interaction. All the constituent average energies of electrostatic interactions occurring between the beads representing charged monomers and ions, and the total energy of electrostatic interactions in the system, were determined from Coulomb's equation, assuming that all the objects were point electric charges:

$U_{\mathrm{M}}=\frac{b}{4 \pi \varepsilon_{o} \varepsilon} \sum_{\mathrm{i}} \sum_{\mathrm{j}} \frac{q_{\mathrm{i}} q_{\mathrm{j}}}{e^{2} r_{\mathrm{i}, \mathrm{j}}}$ 
where $\varepsilon_{0}$ is the permittivity of vacuum and $\varepsilon$ denotes relative permittivity of the medium, whereas $q_{\mathrm{i}}$ and $q_{\mathrm{j}}$ represent electric charges of the currently analyzed charged objects located at the distance $r_{\mathrm{ij}}$. The computation was performed using the minimum image convention and the cutoff radius equal to half box length $(300 b)$ in the direction parallel to the nanochannel. In most simulations $(N=50, \sigma=0.2)$, the radius applied guaranties that only long-range ionic interactions of the energy smaller than 0.03 the elementary interaction between neighboring charged polymer beads were neglected.

Within the framework of the model, it was assumed that $\varepsilon_{0} b / e$ was equal to $8 \cdot 10^{-4}$, which ensured that the energy of interactions between two monovalent point charges located at the distance $b$ (i.e., in the immediate vicinity determined by the lattice constant) was equivalent to $100 k_{\mathrm{B}} T$ (since for so small distance $\varepsilon=1$ ). The energy of charged beads in the external electric field was calculated from the equation:

$U_{\mathrm{E}}=\frac{E}{\varepsilon_{o} \varepsilon} \sum_{\mathrm{i}} \frac{q_{\mathrm{i}} \mathrm{x}_{\mathrm{i}}}{e b}$

where $E$ is the external electric field directed in the same way in all unit boxes (usually, $E\left(e b / k_{\mathrm{B}} T\right)=0.1$ ) and $x_{\mathrm{i}}$ is the distance of the $i$-th charged bead from the bottom of the nanochannel. The influence of dielectric nanochannel walls on the movements of electrically charged species was neglected.

Calculations of the conformational entropy of the chains were performed using the modified SCM (statistical counting method) [54] from the equation:

$\frac{S_{\mathrm{N}}}{k_{\mathrm{B}}}=\sum_{i=1}^{N-1} \ln \left(\omega_{i}\right)$

where $N$ denotes the number of beads in the chain, $k_{\mathrm{B}}$ is the Boltzmann constant, and $\omega_{i}$ is the effective coordination number of each succeeding bead in the chain, which is simply equal to the number of lattice sites occupied by the solvent molecules. The modification consists in application of the "phantom chain"; initially the whole chain was removed from the simulation space, than-following the chain path-the $\omega_{i}$ value was calculated step-by-step. After each step, the succeeding bead was put back to the chain structure.

The entropy contribution $S_{\mathrm{E}}$ due to the rearrangement of ions ( $B_{\mathrm{I}}$ beads) in the nanochannel was calculated as the difference between the mixing entropies of beads I in the simulation box in the presence and in the absence of the polymer chains:

$\frac{S_{\mathrm{E}}}{k_{\mathrm{B}}}=-4 \pi \sum_{i=1}^{R_{\mathrm{S}}} i^{2}\left(\rho_{-} \ln \left(\rho_{-}\right)+\rho_{+} \ln \left(\rho_{+}\right)-\rho_{0} \ln \left(\rho_{0}\right)\right)$

where $\rho_{-}$and $\rho_{+}$denote densities of anions and cations, respectively, along the channel, whereas $\rho_{0}$ stands for the density distribution of the corresponding kind of beads I in the box without charged chains, assuming that at each point of the simulation the stationary state of counterion cloud is reached. The densities are defined as the ratios of the number of nodes occupied by ions to the total number of nodes in layers around the center of $\mathrm{C} 1$ macromolecule and in the empty channel. The summation radius $R_{\mathrm{S}}$ was equal to $300 \mathrm{~b}$ and satisfied the minimum image convention requirement.

The Helmholtz free energy $A$ of the system was calculated as the sum of (i) energetic contributions $U_{\mathrm{M}}$ and $U_{\mathrm{E}}$ associated with the mutual electrostatic interactions between all charged species in the system and their interaction with the external electric field and (ii) entropic contributions $S_{\mathrm{P}}$ and $S_{\mathrm{E}}$ associated with the chain conformations and the distribution of ions near polymer chains, respectively:

$A=U_{\mathrm{M}}+U_{\mathrm{E}}-T\left(S_{\mathrm{P}}+S_{\mathrm{E}}\right)$

The nMC [48] method was applied for the study of macromolecule translocation and ion migration. The elementary micromodification applied in the simulation consists of (i) random selection of the object to be moved (a polymer bead of one of macromolecules or a simple ion), (ii) a shift of the object to a new position (using elementary VerdierStockmayer algorithms which include the kink-jump, crankshaft (two segments), reptation (bilocal), or end movements $[49,50]$ of a polymer bead or a single jump to one of the neighboring lattice sites of a simple ion), (iii) checking whether the new position does not violate the topological constraints or excluded volume condition, and finally (iv) the Metropolis criterion. In the criterion, umbrella sampling [48] based on the entropies of macromolecules and ions (Eqs. 4 and 5) was applied as a measure of the probability of state of the system, whereas the system energy defined by Eqs. 2 and 3 was used to calculate the Boltzmann factor in the standard Metropolis algorithm at a constant reduced temperature $T=1$. All types of elementary micromodifications were employed with the frequency proportional to the number of objects to which they can be applied because such a procedure provided the correct time scale of the simulation $[55,56]$.

Each simulation started with the macromolecules initially equilibrated and then located at the initial distance between their mass centers approximately equal to $50 \mathrm{~b}$. As a result of the equilibration, the coordinates of mass centers and the distances between them in general were not integers but this uncertainty did not matter since the time from the start of simulation to the moment of macromolecules collision was not essential in the study. The polyion was located always at the opposite side relative to the attractive electrode. Then, the electrical field was switched on and directed movement of polyion (C1) started.

All the results presented in the paper are averages of at least 500-5000 trajectories of pairs of migrating chains. The 
number of beads in both chains tested was $N=10 \div 200$ (in most simulations $N=50$ ). Each single simulation is a result up to $10^{5} \mathrm{nMC}$ steps (one nMC step corresponds to the number of shifts needed to give each of the beads the possibility to move once, i.e., 100 elementary macromolecule micromodifications of two macromolecules of $N=50$ ). The actual number of steps was limited by reaching the bottom of the nanochannel by molecule $\mathrm{C} 1$. The unit of the corresponding time period was defined as one nMC cycle.

The data collected from the simulation contain the following: positions of mass centers of coils, all components of free energy of the system including internal energy of electrostatic interaction between all charged species - ions and charged segments, the electrostatic interaction of the species with the external electric field, conformational entropy of polyion and uncharged macromolecule, and configurational entropy of ions of a simple electrolyte in a dispersion medium. Since the athermal conditions were assumed, the study neglects all other intermolecular interactions as well as interaction with nanochannel walls except for the excluded volume effect. It should also be stressed that the studied system represents a damping dynamic model, holding only the activation aspect of diffusion and neglecting of the inertia effects [57].

\section{Results}

\section{Details of motions of the polyion and uncharged macromolecule}

The studies of the behavior of macromolecular pairs, consisting of a polyelectrolyte and a nonionic polymer confined in a nanopore, have shown that the translocation of the polyion caused by electric field may induce the collision between macromolecules, their mutual movement, compression of neutral macromolecule, and finally swapping of positions of both macromolecules. The exemplary trajectories of both macromolecules are presented in Figs. 2 and 3.

Figure 2 presents evolution of the system composed of two linear macromolecules in a nanochannel and subjected to an external electric field. One of the macromolecules is electrically charged $(\mathrm{C} 1)$, while the other is neutral $(\mathrm{C} 2)$. The trajectories of the centers of mass of both macromolecules can be divided into a few sections corresponding to the stages of the processes taking place in the nanochannel: (1) mutual approach of $\mathrm{C} 1$ and $\mathrm{C} 2$ ended with a collision, (2) stage of contact and permeation of $\mathrm{C} 2$ by $\mathrm{C} 1$ (induction time), (3) swapping of positions of $\mathrm{C} 1$ and $\mathrm{C} 2$ centers, (4) segregation of both macromolecules, and (5) diffusion of the released $\mathrm{C} 2$ into the bulk of nanochannel. The main parameters determining the rate of the whole process studied here are the time of swapping induction $t_{\mathrm{S}}$, i.e., the time period between the collision $\left(t_{1}\right)$ and exchange of coils' positions $\left(t_{2}\right)$ and the time of segregation $t_{\mathrm{R}}$, i.e., the time between swapping $\left(t_{2}\right)$ and final release $\left(t_{3}\right)$ of macromolecules. The moment of collision of the macromolecules $\left(t_{1}\right)$ was assumed to be the time when the mass centers of both macromolecules approach each other to a distance equal to the double radius of gyration (more specifically to the radius of gyration of the uncharged macromolecule in the bulk, $\left.R_{\mathrm{g} 0}\right)$. The moment of swapping $\left(t_{2}\right)$ was precisely specified by the change in the sign of the vector projection on the axis parallel to the channel, representing the distance between the positions of macromolecules' centers, namely it is a moment of passing by the centers of mass. The moment of macromolecule release $\left(t_{3}\right)$ was taken as the time when they cross-up the distance of $R_{\mathrm{g} 0}$ moving away from each other.

The evolution of macromolecules can be more complex than that shown in Fig. 2. When the stimulus of the process, the electric field strength, is small, the swapping becomes a reversible process and "oscillations" of both macromolecules in the course of their travel along the nanochannel occur. Figure 3 presents the visualization of collision events in the course of migration of molecules. The collisions may result in bouncing when the chains come close to each other and then return in the direction they came from or in the swapping of their positions. In most simulations presented in the study (at $\left.E\left(e b / k_{\mathrm{B}} T\right)=0.1\right)$, the bouncing and multiple swapping events were negligible.

The evolution of molecule conformation is well illustrated by the ratio of the $x$ component of the radius of gyration to the nanochannel width $2 R_{\mathrm{g}}(x) / D$ (Fig. 4). As shown, initially both conformations are usually elongated along the $x$-axis and undergo strong fluctuation. The initial elongation of $\mathrm{C} 1$ molecule is relatively large and rather constant as a result of electrostatic repulsion. C2 molecule structure is less stiff and undergoes stronger fluctuation. After the collision, the fluctuations of elongation of both macromolecules are much weaker since the number of available lattice nodes is reduced by beads of the other chain. The elongation of $\mathrm{C} 1$ molecule along the nanochannel is still somewhat larger than that of $\mathrm{C} 2$ because of the electric repulsion that stretches the conformation, which is not screened by any species and only slightly influenced by the counterion cloud. The values of $2 R_{\mathrm{g}}(x) / D$ of $\mathrm{C} 2$ molecule after collision are close to 1 , which indicates that if forms a plug clogging the nanochannel and pushed by $\mathrm{C} 1$ molecule. Near the swapping moment, the spectacular changes in the conformations of interpenetrating molecules are observed$\mathrm{C} 2$ molecule collapses whereas the $\mathrm{C} 1$ polyion takes more elongated structure. Finally, in the course of segregation and the conformation releasing stage, both molecules are heavily stretched along the nanochannel. After the separation stage, in the course of the following free diffusive expansion, a significant increase in the elongation of $\mathrm{C} 2$ molecule takes place whereas $\mathrm{C} 1$ undergoing the electric interaction remains compressed at the bottom of the nanochannel. 
Fig. 2 Exemplary trajectories of two macromolecules (the distances of both macromolecules from the bottom of the nanochannel versus the number of nMC steps $I$ ), a polyion and an electrically neutral macromolecule, confined in a nanopore and subjected to an electric field. The figure presents the stages of motion of the neutral macromolecule $\mathrm{C} 2$ induced by translocation of the polyion in the electric field: diffusion, collision with macromolecule $\mathrm{C} 1$ (1), forced translocation, compression, swapping position with $\mathrm{C} 1$ (2), segregation to the distance of the radius of gyration (3), and finally free diffusion $(N=50, D=5 b)$

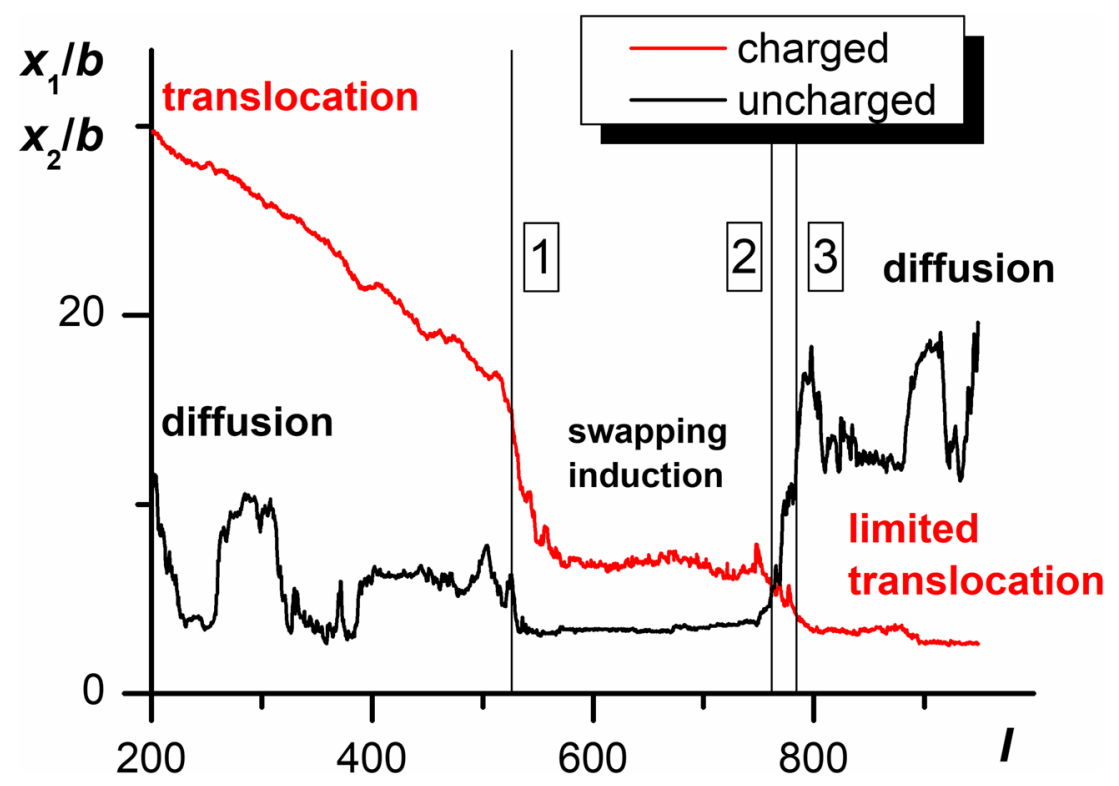

The evolution of the conformational entropy of both molecules is shown in Fig. 5. As shown, the initial entropies of $\mathrm{C} 1$ and $\mathrm{C} 2$ are quite large. The $\mathrm{C} 2$ entropy undergoes higher fluctuations since the molecule conformation is more chaotic and not stretched by the electrostatic repulsion. The entropy of $\mathrm{C} 1$ is rather constant, which corresponds to a constant shift of the whole practically undeformed molecule induced by the external force. After collision, both entropies dramatically decrease. The entropy of $\mathrm{C} 1$ is larger than that of the compressed $\mathrm{C} 2$ molecule. In this stage, both molecules interpenetrate each other, so the available space is reduced by neighbors. The separation following the swapping of molecules produces an increase in the conformation entropy of both of them. The increase in entropy of $\mathrm{C} 2$ is much higher-the final entropy is similar to that at the beginning of the process. The entropy of $\mathrm{C} 2$ is reduced by the compression induced by electrostatic attraction to the bottom of the nanochannel.

As shown in Fig. 6, the energy of interaction of the polyion with the external electric field, which dominates in both the energy and the entropy components of the free energy of the system, can be approximately represented by straight lines whose slopes are determined by the location of $\mathrm{C} 1$ molecule in the course of the whole process. Initially, a fast decrease in free energy results from the relatively fast electrophoresis of the polyion. The collision with $\mathrm{C} 2 \mathrm{~mol}-$ ecule breaks this fast motion - both molecules in the course of interpenetration move very slowly or even stop until the moment of swapping, when the $\mathrm{C} 1$ molecule velocity
Fig. 3 Difference between mass center coordinates of migrating macromolecules versus the number of nMC steps at the weak electric field $(N=50, D=5 b$, $\left.E\left(e b / k_{\mathrm{B}} T\right)=0.1\right)$

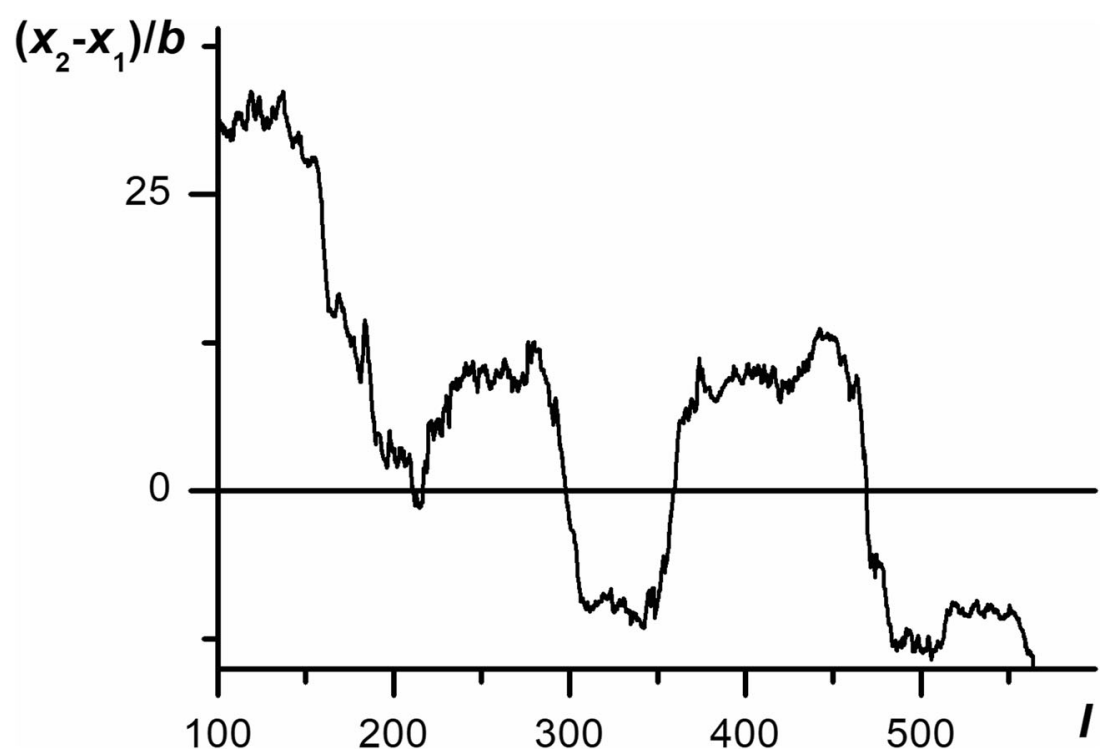


Fig. 4 Evolution of the ratio of the $x$ component of the diameter of gyration to the nanochannel width $\left(2 R_{\mathrm{g}}(x) / D\right)$ of both macromolecules in the course of migration specified by the number of nMC steps, $I$, accompanying the evolution shown in Fig. $2(N=50, D=5 b)$

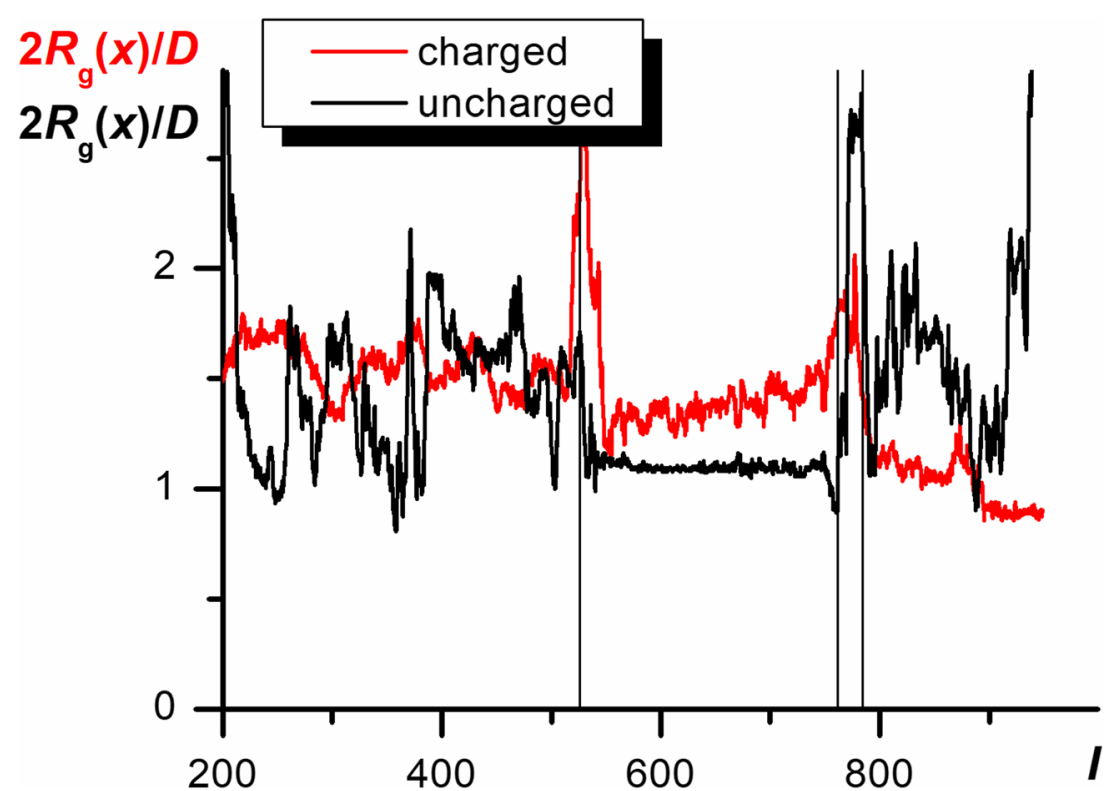

increases. This stage of the $\mathrm{C} 1$ motion is the fastest since the electrophoresis of macromolecule is assisted by entropophoresis caused by the increase in the conformational entropy of $\mathrm{C} 1$ and $\mathrm{C} 2$. Finally, the free energy of the system takes a minimum value when $\mathrm{C} 1$ molecule touches the nanochannel bottom. The position of molecule $\mathrm{C} 2$ does not bring a direct contribution to the free energy of the system because it is represented by electrically uncharged chain in athermal solution.

The exemplary results presented in Figs. 2, 3, 4, 5, and 6 were obtained by means of single simulations. Further results are averages of independent 500-5000 simulations.
Figure 7 shows the time distributions of particular steps of the studied process. The wide distribution of the time needed for macromolecule collision is not very representative of the macromolecule properties since its width depends on the arbitrarily assumed initial positions of both molecules. The time distributions of the following stages - the incubation time of swapping (interpenetration $\mathrm{C} 2$ by $\mathrm{C} 1$ ) and the time of final segregation shown in Fig. 7-are much narrower and can be well approximated by the logarithmic normal distribution. The process of mutual releasing of molecules (segregation) is faster than interpenetration since it is
Fig. 5 Dependence of conformational entropies of both macromolecules on the number of nMC steps in the course of migration accompanying the evolution shown in Fig. 2 ( $N=50$, $D=5 b$ )

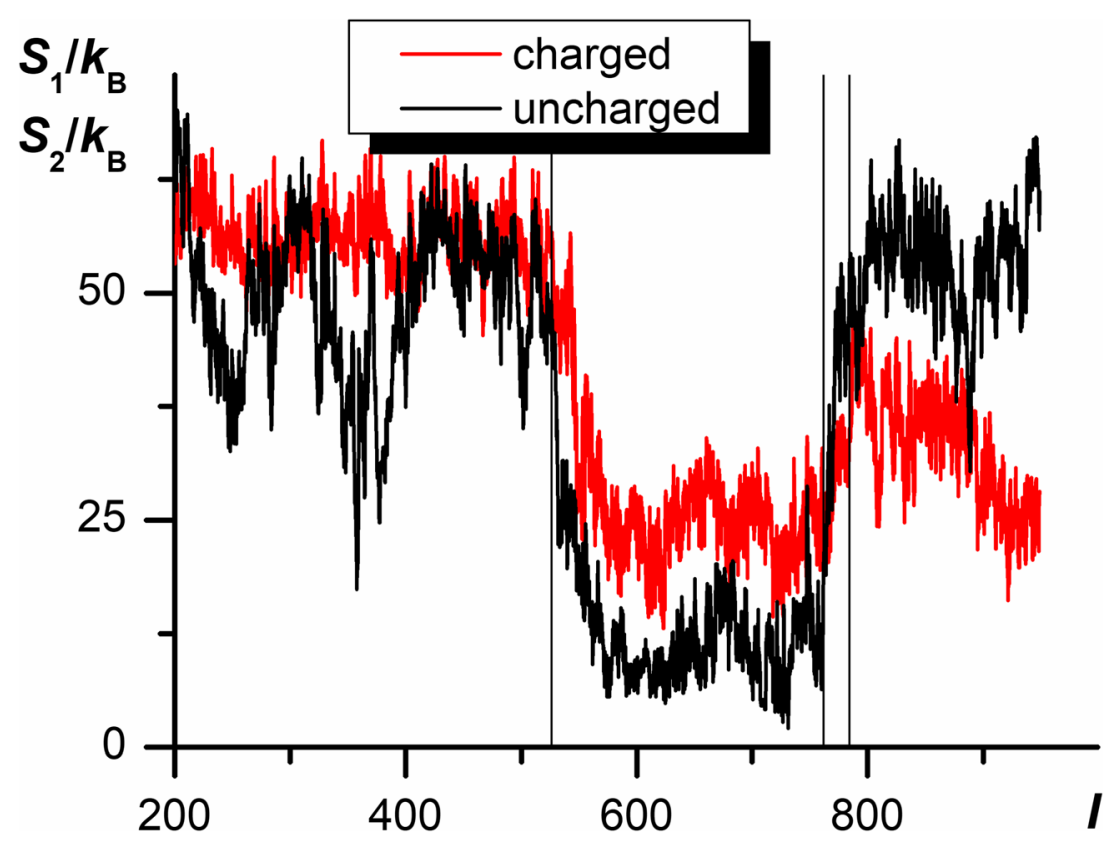


Fig. 6 The decrease in the total free energy of the system on the number of nMC steps in the course of migration accompanying the evolution shown in Fig. $2(N=50, D=5 b)$

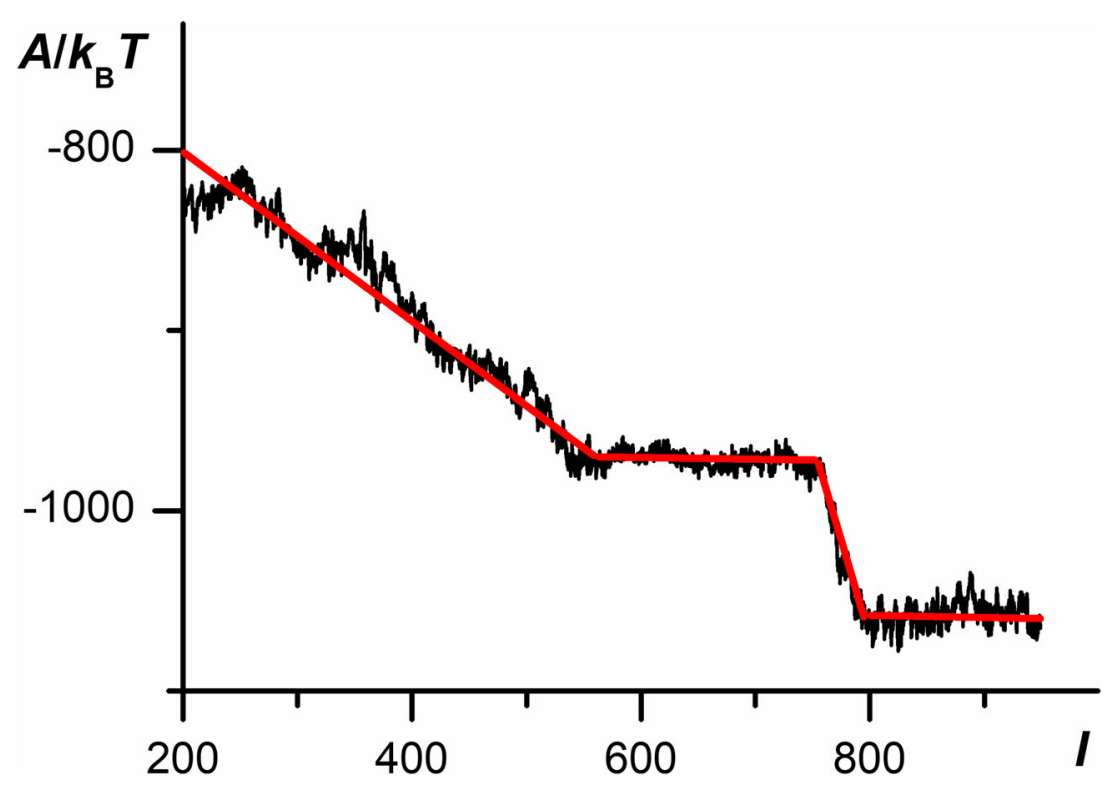

triggered by two consistent phenomena: electrophoresis and entropophoresis.

\section{Generalization of the system behavior-some scaling laws}

The results collected below are the attempt at quantitative description of characteristic time or rate of selected stages of the studied process. According to the relaxation of single macromolecules [48, 58, 59] or segregation of pairs of macromolecules $[33,35]$ in the tight confinement, we postulated the universal scaling law linking the time of the stage $t$ with the number of beads in the polymer chain $N$ and the width of the nanochannel $D$ :

$t=a N^{v} D^{\mu}$

where $a$ is the proportionality coefficient.

As shown in Fig. 8a, an increase in the chain length causes an increase in the incubation time $t_{\mathrm{S}}$. The relation between $t_{\mathrm{S}}$ and the number of beads is linear in the logarithmic system of coordinates. The mean value of the slope at $E\left(e b / k_{\mathrm{B}} T\right)=0.1$ is $v=1.50 \pm 0.12$, which seems to be close to the time of relaxation of a single macromolecule in a confining channel $(v=$ $1.6 \pm 0.04$ or $1.33[48,60])$. However, the process presented
Fig. 7 Distributions of time (expressed by the number nMC steps) needed for macromolecule swapping and releasing $(N=50$, $D=5 b$ ). The fitted log-normal distributions are marked in blue

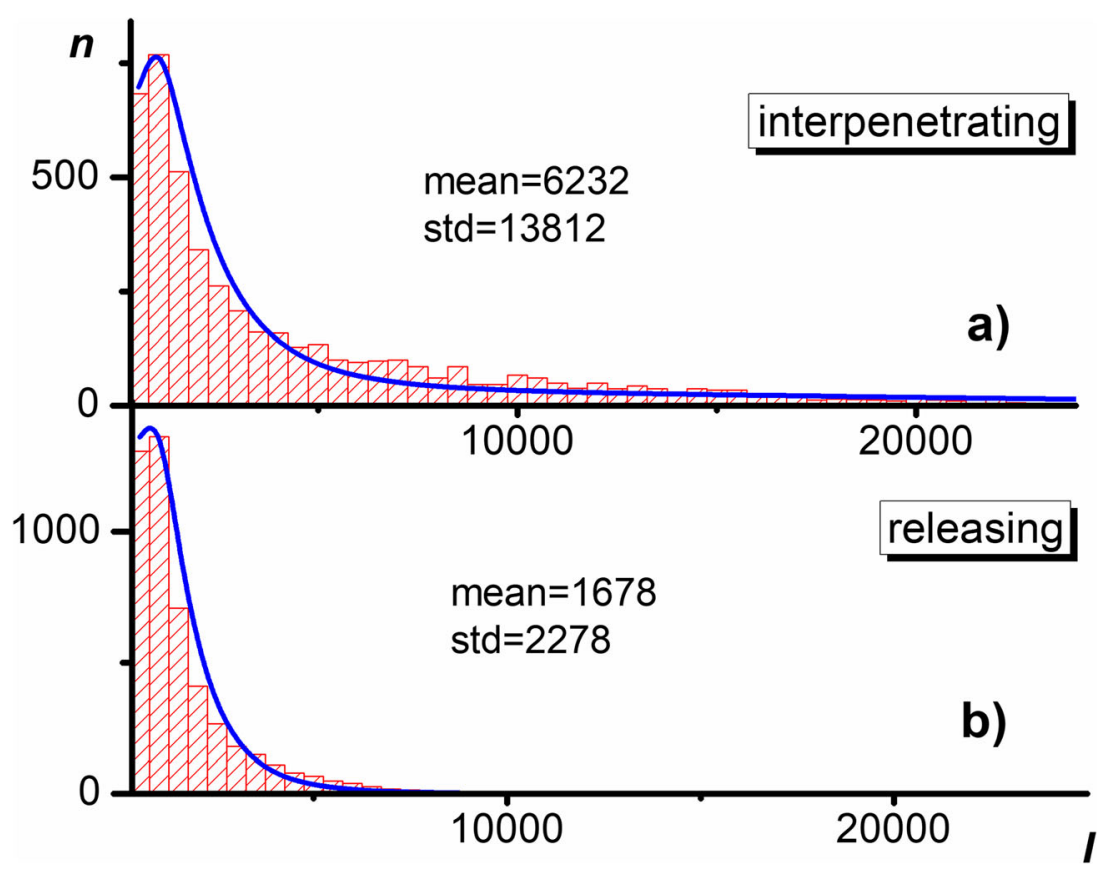


Fig. 8 The mean swapping induction time (a) and segregation time (b) versus the number of beads in the chain $(D=5 b)$

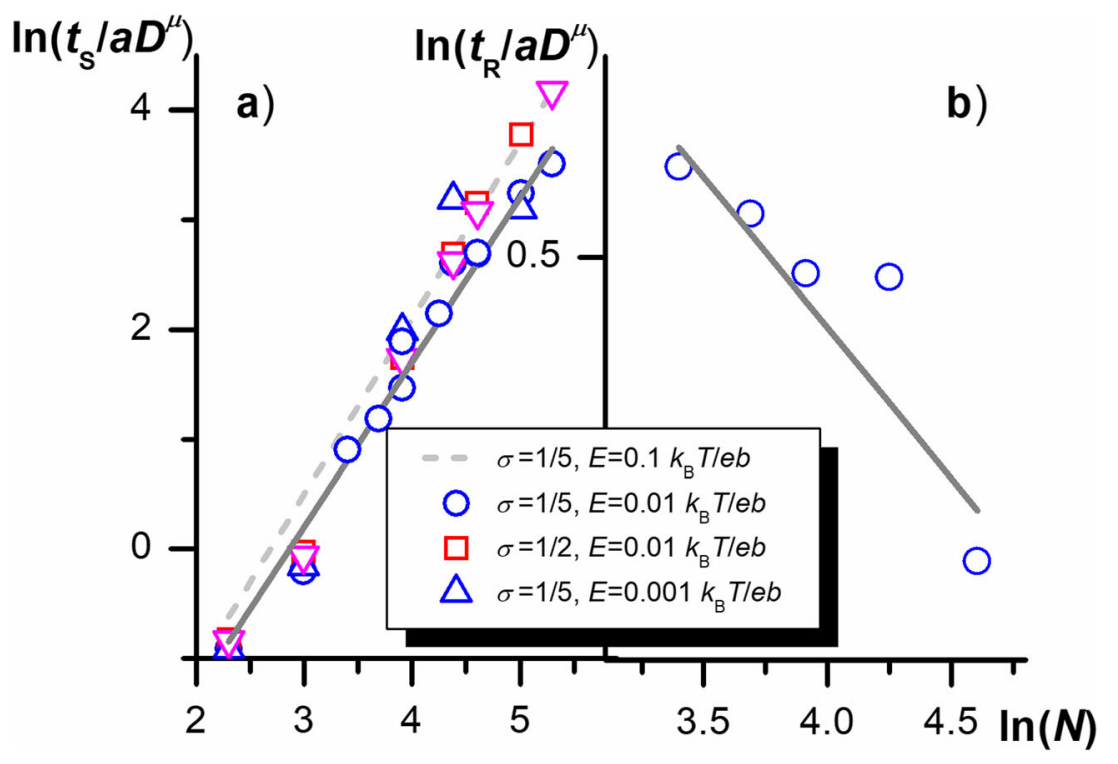

here is not a pure segregation nor a relaxation on the individual macromolecule, but it is a superposition of the pushing and interpenetration of macromolecule $\mathrm{C} 2$ by $\mathrm{C} 1$. The results obtained for weaker external electric field $\left(E\left(e b / k_{\mathrm{B}} T\right)=0.01\right.$ and $0.001)$ are slightly biased producing a higher slope $(v \approx 1.6$, marked in dashed line). However, the longer swapping induction time results from repeated bouncing of macromolecules before swapping (as shown in Fig. 3).

The time of segregation process $t_{\mathrm{R}}$ versus number of beads scales approximately with the exponent $v=-0.3 \pm 0.1$ (see Fig. 8b) showing the increase in mutual velocity of macromolecules with the number of their beads. This dependence seems to be the opposite of that observed for the systems with no external electric field applied $(v=0.3[33,35])$ and should be related to the relatively large electrostatic force pulling out $\mathrm{C} 1$ macromolecule from the $\mathrm{C} 2$ coil.
Dependencies of characteristic times of swapping and segregation processes on the nanochannel width are presented in Figs. 9a and 10a. As shown, both times $t_{\mathrm{S}}$ and $t_{\mathrm{R}}$ scale well with $D$, however with different exponents: $\mu=1.5 \pm 0.09$ (for swapping) and $\mu=2.0 \pm 0.11$ (for segregation). The difference is due to the fact that the swapping and segregation processes differ in some aspects: while swapping is influenced by two opposite forces induced by electrical field and the decrease in the conformational entropy, in the segregation both forces cooperate. However, these phenomena are not symmetrical since the relaxation of the $\mathrm{C} 1$ structure is often influenced by an additional confinement - the bottom of the nanochannel.

The unexpected increase in the incubation time of swapping with $D$ can be explained if one takes into account that
Fig. 9 The mean swapping induction time (a) and the mean mutual velocity of chains in the time period between collision and swapping (b) versus the nanochannel width $(N=50)$

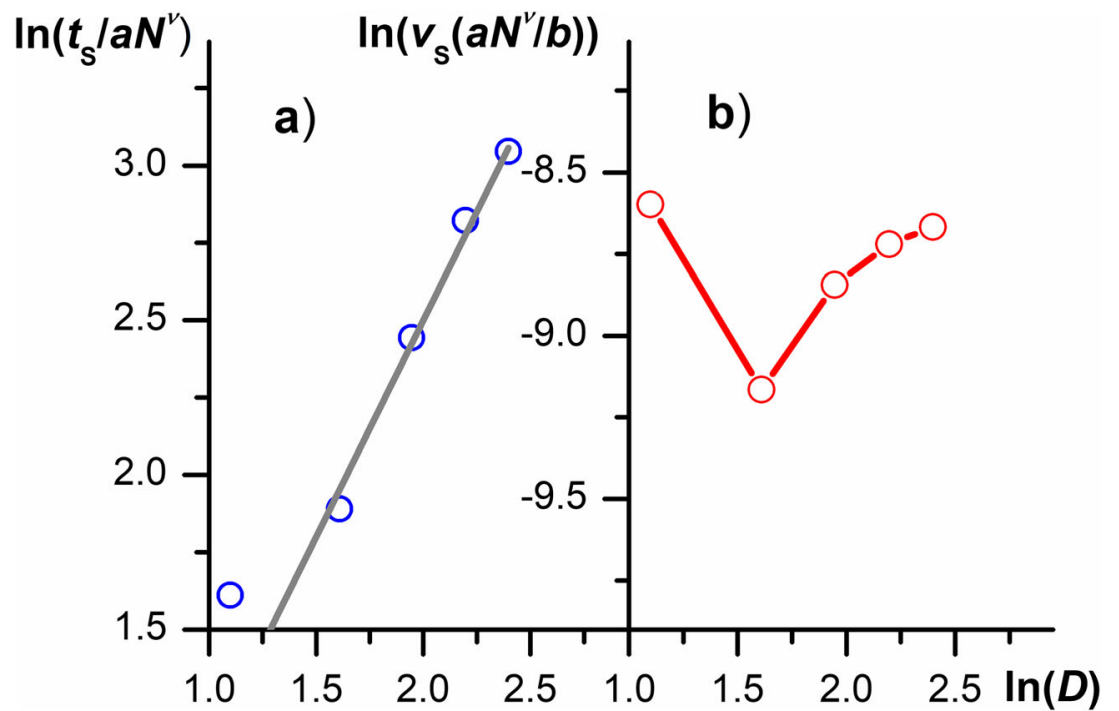


Fig. 10 The mean segregation time (a) and the mean mutual velocity of chains in the time period between swapping and releasing of the chain $\mathrm{C} 2$ (b) versus the nanochannel width $(N=50)$

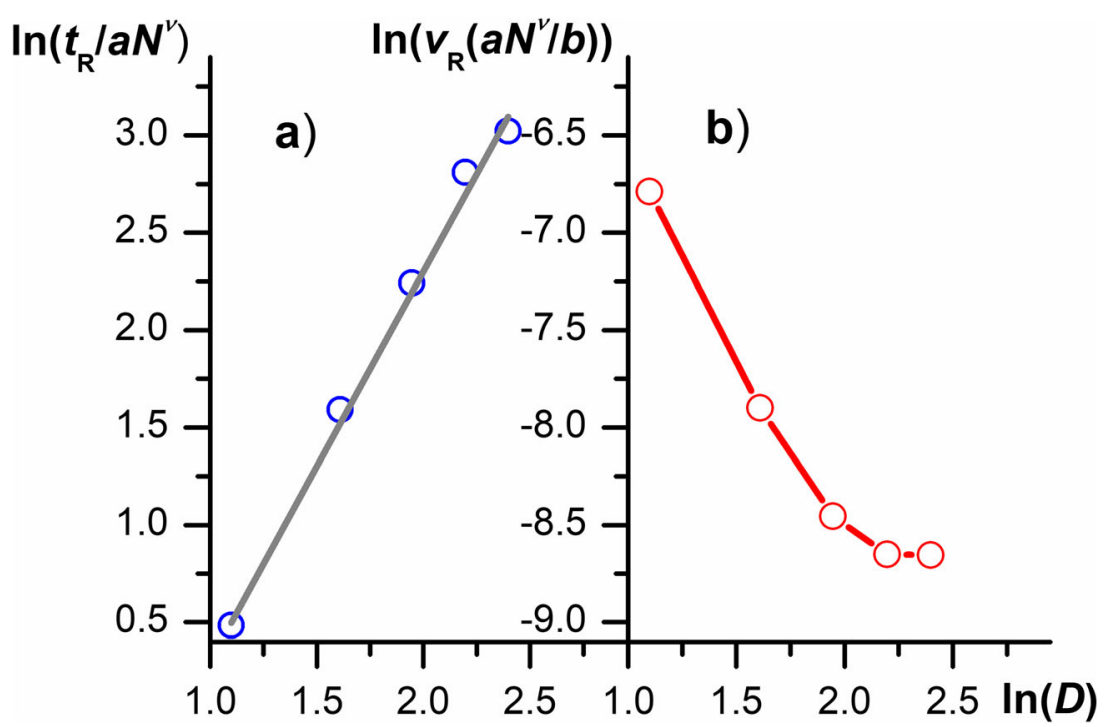

not only the time but also the distance covered by macromolecules in the course of pushing of $\mathrm{C} 2$ molecule by $\mathrm{C} 1$ and simultaneous interpenetration of $\mathrm{C} 2$ by $\mathrm{C} 1$, depend on the pore diameter. The mutual velocity (the velocity of $\mathrm{C} 1$ relative to molecule $\mathrm{C} 2$ ) $v_{\mathrm{S}}$ versus $D$ shown in Fig. 9b, although slightly irregular, illustrates a more intuitive trend-an increase in nanochannel width induces an increase in $v_{\mathrm{S}}$.

The dependence of the segregation time on the nanochannel width presented in Fig. 10a is less controversial and generally agrees with the relationship obtained for pure segregation reported in $[33,35]$ (where $\mu=0.3$ ) since the $t_{\mathrm{R}}$ is an increasing function of $D$, however with the exponent $\mu=$ 2.0. The increase in $D$ exerts a diminishing effect on conformational entropy; therefore, the entropic force causing the expansion of macromolecules decreases and the relaxation time increases. However, the electrostatic force pulling $\mathrm{C} 1$ macromolecule can affect $t_{\mathrm{R}}$. Moreover, one should take into account that the distance covered in the segregation stage can be influenced by the bottom of nanochannel which stops the motion of $\mathrm{C} 1$ polyion. These additional effects are probably responsible for the difference in the $\mu$ exponents observed here and in $[33,35]$. Finally, the more adequate relationship of the mutual segregation velocity versus pore width (Fig. 10b) shows that the diminishing trend disappears at large nanochannel widths.

In all simulations presented in the study, the persistent length of chains [61] $(\sim 2 b)$ was smaller than $D$, whereas the radius of gyration in the bulk exceeded $D$ and varied from $8 b$ to about $25 \mathrm{~b}$. It implies rather the de Gennes or transition regime of confinement than the Odijk one [60]. Figures 8, 9, and 10 seem to present reasonable dependencies similar to the results presented for segregation or relaxation of macromolecules in similar circumstances [33-35]. However, the dependencies deal with the special case when the diffusion and forced motion occur at the same time and cannot be compared directly with available experiments and theories.

\section{Conclusions}

It was shown that the polyion moving in the external electric field in the tight channel is able to force the motion of an uncharged macromolecule. After the moment of collision of both molecules, the pushing of the neutral macromolecule accompanied by its interpenetration by the polyion takes place. The interpenetration is associated with changes in conformation of the macromolecules as well as in their entropies and internal energies. In the course of interpenetration, the swapping of mass centers occurs and then, when the electric external field is strong enough, the unidirectional segregation takes place, in which the uncharged macromolecule diffuses to the bulk of the nanochannel. The induction time of swapping scales with the number of beads in polymer chains in the way independent of the density of electric charges on the macromolecule and the strength of electric field.

The results presented permit designing new methods for manipulation and control of individual macromolecules subjected to external electric field. The hypothetical system of nanochannels and/or microswitches consisting of a labyrinth of crossing patches switched on by electrophoretic or entropic gradients seems to be able to be used for the segregation/isolation of macromolecules of assumed properties. The system can be controlled by the external electric field, $\mathrm{pH}$, and the ionic strength of dispersion medium.

However, it should be noted that since the simulations concern a relatively small number of ionic beads, the presented results are applicable only to the systems containing an electrolyte solution of extremely small ionic strength. 
Open Access This article is distributed under the terms of the Creative Commons Attribution 4.0 International License (http:// creativecommons.org/licenses/by/4.0/), which permits unrestricted use, distribution, and reproduction in any medium, provided you give appropriate credit to the original author(s) and the source, provide a link to the Creative Commons license, and indicate if changes were made.

\section{References}

1. Neuman KC, Lionet T, Allemend J-F (2009) Single-molecule micromanipulation techniques. Annu Rev Mater 37:33-67. https:// doi.org/10.1146/annurev.matsci.37.052506.084336

2. Wang FF, Sam L, Liu L, Lee GB, Li WJ (2015) Micro and nano manipulation and assembly by optically induced electrokinetics in micro- and nanomanipulation tools. In: Sunand Y, Liu X (eds). Wiley VCH Weinheim, Germany

3. Ishijima A, Kojima H, Higuchi H, Harada Y, Funatsu T, Yanagida T (1996) Multiple- and single-molecule analysis of the actomyosin motor by nanometer-piconewton manipulation with a microneedle: unitary steps and forces. Biophys J 70:383-400. https://doi.org/10. 1016/S0006-3495(96)79582-6

4. Gosse C, Croquette V (2002) Magnetic tweezers: micromanipulation and force measurement at the molecular level. Biophys J 82: 3314-3329. https://doi.org/10.1016/S0006-3495(02)75672-5

5. Ludwig M, Rief M, Schmidt L, Li H, Oesterhelt F, Gautel M, Gaub HE (1999) AFM, a tool for single-molecule experiments. App Phys A 68:173-176. https://doi.org/10.1007/s003390050873

6. Holland NB, Hugel T, Neuert G, Cattani-Scholz A, Renner C, Oesterhelt D, Moroder L, Seitz M, Gaub HE (2003) Single molecule force spectroscopy of azobenzene polymers: switching elasticity of single photochromic macromolecule. Macromolecules 36: 2015-2023. https://doi.org/10.1021/ma021139s

7. Neuman KC, Block SM (2004) Optical trapping. Rev Sci Instrum 75(9):2787. https://doi.org/10.1063/1.1785844

8. Jülicher F, Ajdari A, Prost J (1997) Modeling molecular motors. Rev Mod Phys 69(4):1269-1281. https://doi.org/10.1103/ RevModPhys.69.1269

9. MacDonald MP, Spalding GC, Dholakia K (2003) Microfluidic sorting in an optical lattice. Nature 426(6965):421-424. https:// doi.org/10.1038/nature02144

10. Austin R (2003) Nanopores: the art of sucking spaghetti. Nat Mater 2(9):567-568. https://doi.org/10.1038/nmat962

11. Cohen AE (2005) Control of nanoparticles with arbitrary twodimensional force fields. Phys Rev Lett 94(11):118102. https:// doi.org/10.1103/PhysRevLett.94.118102

12. Wong PK, Wang TH, Deval JH, Ho CM (2004) Electrokinetics in micro devices for biotechnology applications. IEEE-ASME Trans Mechatron 9(2):366-376. https://doi.org/10.1109/TMECH.2004. 828659

13. Chiou PY, Ohta AT, Wu MC (2005) Massively parallel manipulation of single cells and microparticles using optical images. Nature 436(7049):370372. https://doi.org/10.1038/nature03831

14. Lin YH, Lee GB (2008) Optically induced flow cytometry for continuous microparticle counting and sorting. Biosens Bioelectron 24(4):572-578. https://doi.org/10.1016/j.bios.2008.06.008

15. Mizuno A, Nishioka M, Ohno Y, Dascalescu LD (1995) Liquid microvortex generated around a laser focal point in an intense high-frequency electric field. IEEE Trans Ind Appl 31(3):464468. https://doi.org/10.1109/28.382104

16. Jamshidi A, Neale SL, Yu K, Pauzauskie PJ, Schuck PJ, Valley JK, Hsu HY, Ohta AT, Wu MC (2009) NanoPen: dynamic, low-power, and light-actuated patterning of nanoparticles. Nano Lett 9(8): 2921-2925. https://doi.org/10.1021/n1901239a
17. Ramos A, Morgan H, Green NG, Castellanos A (1999) AC electricfield-induced fluid flow in microelectrodes. J Colloid Interface Sci 217(2):420-422. https://doi.org/10.1006/jcis.1999.6346

18. Islam N, Lian M, Wu J (2007) Enhancing microcantilever capability with integrated AC electroosmotic trapping. Microfluid Nanofluid 3(3): 369-375. https://doi.org/10.1007/s10404-006-0138-z

19. Pohl HA (1978) Dielectrophoresis : the behavior of neutral matter in nonuniform electric fields. Cambridge University Press, Cambridge

20. Sikorski A, Żukowska I (2008) Dynamics of branched chain solution in adsorbing slit. A Monte Carlo study. Rheol Acta 47(5):571577. https://doi.org/10.1007/s00397-007-0241-4

21. Stavis SM, Geist J, Gaitan M, Locascio LE, Strychalski EA (2012) DNA molecules descending a nanofluidic staircase by entropophoresis. Lab Chip 12(6):1174-1182. https://doi.org/10. 1039/c2lc21152a

22. Stavis SM, Strychalski EA, Gaitan M (2009) Nanofluidic structures with complex three-dimensional surfaces. Nanotechnology 20(16): 165302. https://doi.org/10.1088/0957-4484/20/16/165302

23. Romiszowski P, Sikorski A (2012) Motion of star-branched chains in a nanochannel. A Monte Carlo study. Comput Methods Sci Technol 18:39-44. https://doi.org/10.12921/cmst.2012.18.01.3944

24. Dikin DA, Stankovich S, Zimney EJ, Piner RD, Dommett GHB, Evmenenko G, Nguyen ST, Ruoff RS (2007) Preparation and characterization of graphene oxide paper. Nature 448(7152):457-460. https://doi.org/10.1038/nature06016

25. Novoselov KS, Fal'ko VI, Colombo L, Gellert PR, Schwab MG, Kim K (2012) A roadmap for graphene. Nature 490(7419):192200. https://doi.org/10.1038/nature11458

26. Joshi RK, Alwarappan S, Yoshimura M, Sahajwalla V, Nishina Y (2015) Graphene oxide: the new membrane material. Appl MaterToday 1(1):1-12. https://doi.org/10.1016/j.apmt.2015.06.002

27. Yoon HW, Cho YH, Park HB (2016) Graphene-based membranes: status and prospects. Philos Trans R Soc A 374:20150024. https:// doi.org/10.1098/rsta.2015.0024

28. Yoshida H, Bocquet L (2016) Labyrinthine water flow across multilayer graphene-based membranes: molecular dynamics versus continuum predictions. J Chem Phys 144(23):234701. https://doi. org/10.1063/1.4953685

29. Nair RR, Wu HA, Jayaram PN, Grigorieva IV, Geim AK (2012) Unimpeded permeation of water through helium-leak-tight graphene-based membranes. Science 335(6067):442-444. https:// doi.org/10.1126/science.1211694

30. Cheng C, Jiang G, Garvey CJ, Wang Y, Simon GP, Liu JZ, Li D (2016) Ion transport in complex layered graphene-based membranes with tuneable interlayer spacing. Sci Adv 2(2):e1501272. https://doi.org/10.1126/sciadv.1501272

31. Negi S, Chandra A (2018) Ionic switch using nano-channels in polymeric membrane. Ionics 25(3):1123-1130. https://doi.org/10. 1007/s11581-018-2618-z

32. Tagliazucchi M, Szleifer I (2015) Transport mechanisms in nanopores and nanochannels: can we mimic nature? Mater Today 18(3):131-142. https://doi.org/10.1016/j.mattod.2014.10.020

33. Minina E, Arnold A (2015) Entropic segregation of ring polymers in cylindrical confinement. Macromolecules 48(14):4998-5005. https://oi.org/10.1021/acs.macromol.5b00636

34. Nowicki W (2019) Segregation of ring polyelectrolytes in nanochannel. J Chem Phys 150(1):014902. https://doi.org/10.1063/1. 5064512

35. Arnold A, Bozorgui B, Frenkel D, Ha BY, Jun S (2007) Unexpected relaxation dynamics of a self-avoiding polymer in cylindrical confinement. J Chem Phys 127(16):164903. https://doi.org/10.1063/1. 2799513

36. Guo LJ, Cheng X, Chou CF (2004) Fabrication of size-controllable nanofluidic channels by nanoimprinting and its application for 
DNA stretching. Nano Lett 4(1):69-73. https://doi.org/10.1021/ n1034877i

37. Tegenfeldt J, Prinz C, Cao H, Chou S, Reisner W, Riehn R, Wang YM, Cox EC, Sturm JC, Silberzan P, Austin RH (2004) The dynamics of genomic-length DNA molecules in 100-nm channels. Proc Natl Acad Sci U S A 101(30):10979-10983. https://doi.org/ 10.1073/pnas.0403849101

38. Brochard F, de Gennes PG (1977) Dynamics of confined polymer chains. J Chem Phys 67(1):52. https://doi.org/10.1063/1.434540

39. de Gennes PG (1979) Scaling concepts in polymer physics. Cornell University Press, Ithaca

40. Jendrejack RM, Schwartz DC, Graham MD, Pablo JJ (2003) Effect of confinement on DNA dynamics in microfluidic devices. J Chem Phys 119(2):1165. https://doi.org/10.1063/1.1575200

41. Schaefer DW, Joanny FJ, Pincus P (1980) Dynamics of semiflexible polymers in solution. Macromolecules 13(5):1280-1289. https://doi.org/10.1021/ma60077a048

42. Jun S, Mulder B (2006) Entropy-driven spatial organization of highly confined polymers: lessons for the bacterial chromosome. Proc Natl Acad Sci USA 103(33):12388-12393. https://doi.org/10. 1073/pnas.0605305103

43. Arnold A, Jun S (2007) Time scale of entropic segregation of flexible polymers in confinement: implications for chromosome segregation in filamentous bacteria. Phys Rev E 76(3):031901. https:// doi.org/10.1103/PhysRevE.76.031901

44. Kasianowicz JJ (2002) Physics of DNA threading through a nanometer pore and applications to simultaneous multianalyte sensing. In: Kasianowicz JJ, Kellermayer MSZ, Dreamer DW (eds) Structure and dynamics of confined polymers. Kluwer Academic, Dordrecht

45. Smeets RMM, Keyser UF, Krapf D, Wu M-Y, Dekker NH, Dekker C (2006) Salt dependence of ion transport and DNA translocation through solid-state nanopores. Nano Lett 6(1):89-95. https://doi. org $/ 10.1021 / \mathrm{n} 1052107 \mathrm{w}$

46. Saltzman EJ, Muthukumar M (2009) Conformation and dynamics of model polymer in connected chamber-pore system. J Chem Phys 131(21):214903. https://doi.org/10.1063/1.3267487

47. Reisner W, Morton K, Riehn R, Wang YM, Yu Z, Rosen M, Sturm JC, Chou SY, Frey E, Austin RH (2005) Statics and dynamics of single DNA molecules confined in nanochannels. Phys Rev Lett 94(19):196101. https://doi.org/10.1103/PhysRevLett.94.196101

48. Attard P (2012) Non-equilibrium thermodynamics and statistical mechanics: foundations and applications. Oxford University Press

49. Verdier PH, Stockmayer WH (1962) Monte Carlo calculations on the dynamics of polymers in dilute solution. J Chem Phys 36(1): 227. https://doi.org/10.1063/1.1732301
50. Verdier PH (1969) A simulation model for the study of the motion of random-coil polymer chains. J Comput Phys 4:224-210. https:// doi.org/10.1016/0021-9991(69)90067-9

51. Ruckenstein E, Schiby D (1985) Effect of the excluded volume of the hydrated ions on double-layer forces. Langmuir 1(5):612-615. https://doi.org/10.1021/la00065a018

52. Paunov VN, Dimova RI, Kralchevsky PA, Broze G, Mehreteab A (1996) The hydration repulsion between charged surfaces as an interplay of volume exclusion and dielectric saturation effects. J Colloid Interface Sci 182(1):239-248. https://doi.org/10.1006/jcis. 1996.0456

53. Manning GS (1978) The molecular theory of polyelectrolyte solutions with applications to the electrostatic properties of polynucleotides. Q Rev Biophys 11(2):179-246. https://doi.org/10.1017/ S0033583500002031

54. Zhao D, Huang Y, He Z, Qian R (1996) Monte Carlo simulation of the conformational entropy of polymer chains. J Chem Phys 104(4):1672. https://doi.org/10.1063/1.470753

55. Skolnick J, Kolinski A (1990) Dynamics of dense polymer systems: computer simulations and analytic theories. Adv Chem Phys 77: 223-278. https://doi.org/10.1002/9780470141274.ch5

56. Binder K, Müller M, Baschnagel J (2004) Polymer models on the lattice. In: Kotelyanskii M, Theodorou DN (eds) Simulation methods for polymers. Marcel Dekker, NewYork

57. Li J, Ma Y, Hu W (2016) Dynamic Monte Carlo simulation of nonequilibrium Brownian diffusion of single-chain macromolecules. Mol Simul 42(4):321-327. https://doi.org/10.1080/ 08927022.2015.1044454

58. Morse DC (1998) Viscoelasticity of concentrated isotropic solutions of semiflexible polymers. 2. Linear response. Macromolecules 31(2):7044-7067. https://doi.org/10.1021/ ma980304u

59. Maggs AC (1997) Two plateau moduli for actin gels. Phys Rev E 55(6):7396. https://doi.org/10.1103/PhysRevE.55.7396

60. Dorfman KD, Gupta D, Jain A, Muralidhar A, Tree DR (2014) Hydrodynamics of DNA confined in nanoslits and nanochannels. EurPhys J Spec Top 223(14):3179-3200. https://doi.org/10.1140/ epjst/e2014-02326-4

61. Hsu H-P, Paul W, Binder K (2010) Standard definitions of persistence length do not describe the local "intrinsic" stiffness of real polymer chains. Macromolecules 43(6):3094-3102. https://doi.org/ $10.1021 / \mathrm{ma} 902715 \mathrm{e}$

Publisher's note Springer Nature remains neutral with regard to jurisdictional claims in published maps and institutional affiliations. 